\title{
Estimating the effect of semi-transparent low-height road traffic noise barriers with Ultra Weak Variational Formulation
}

\author{
Authors: Lei Ding, Timothy Van Renterghem, Dick Botteldooren
}

Affiliation: Ghent University, Department of Information Technology, Sint-Pietersnieuwstraat 41, B- 9000 Gent, Belgium.

\begin{abstract}
The ultra weak variational formulation (UWVF) approach is used to study the effect of semi-transparent road traffic noise barriers of limited height. This numerical method is extended to simulate sound propagation through a porous medium, based on the Zwicker and Kosten phenomenological porous rigid-frame model. An efficient approach to calculate noise levels in multi-lane road traffic noise situations is presented. The UWVF method was validated successfully by comparison with finite-difference time-domain (FDTD) calculations, for the case of sound propagation near a porous, low-height, and complex shaped noise barrier, and for sound propagation above porous ground in a refracting atmosphere. An assessment is made of the shielding of various porous low-height noise barriers for people on the pavement along the road. Porous barriers were shown to improve noise shielding when compared to geometrically identical rigid noise barriers.
\end{abstract}

\section{Introduction}

The negative effects resulting from exposure to road traffic noise are well-known. The adverse effects of exposure to traffic noise range from annoyance over sleep disturbance, to negative impacts on cognitive functioning and to contribution to cardiovascular diseases (for an overview, see e.g. [1]).

In urban areas, pedestrians on pavements are located very close to road traffic noise sources, and are therefore exposed to very high sound pressure levels. When persons evaluate noise annoyance in surveys, there is evidence that not only the sound levels inside their houses are considered, but also the soundscape in their neighborhood [2]. This means e.g. that adequate façade insulation is not the only answer to the city noise problem.

In urban or suburban areas some common noise reducing measures are hard to apply. A standard noise barrier as found along highways is not appropriate in a city centre, because such constructions are visually too intruding for both pedestrians and car drivers. The use of silent road pavements, on the other hand, is not efficient since the typical vehicle speeds in urban areas are limited. In such situation, engine noise is mostly dominant to rolling noise.

A number of studies have been carried out concerning low-height noise barriers along streets [3, $4,5]$. Optimizing the geometry of such barriers may lead to significant reductions of the noise levels in a zone where pedestrians typically appear [5]. This can be explained by the fact that 
the typical source generation positions of road traffic are located at low heights (ranging from $0.01 \mathrm{~m}$ to $0.75 \mathrm{~m}$ according to the Harmonoise/Imagine road traffic source model [6]), and that such low-height barriers can be placed very close to the traffic lanes.

In this paper, the use of acoustically semi-transparent noise barriers is investigated in 2D. Such barriers naturally have a soft top which is known to be beneficial in reducing grazing sound waves. Furthermore, they reduce reflections towards the other side of the street as well. At low frequencies, the transparency may even lead to conserving some of the positive ground effect. Such barriers are also easy to integrate in a street and they could be visually more pleasing than concrete barriers. These may contain different types of materials like densely packed stones or recycled materials. "Green barriers", consisting of a porous substrate and vegetation, could be interesting as well: green roofs on buildings were found to be very efficient to reduce the intensity of diffracting waves over it $[7,8]$.

Wave-based numerical simulation techniques are needed to accurately design such barriers. Most often, the Boundary Element Method (BEM) is used for this type of calculations [5, 9]. However, BEM fails when the Green's function cannot be obtained, which is typically the case if the properties of the propagation medium are not (piecewise) constant in large areas of the simulation domain. For simulating the situations sketched above, the porous medium might pose a problem when it is not homogeneous. Moreover, to accurately simulate the insertion loss of noise barriers, barrier-induced wind speed gradients should be taken into account $[10,11]$. Volume discretization techniques are therefore preferred.

The finite-difference time-domain (FDTD) technique is a well-validated reference model for complex sound propagation calculations outdoors [11, 12]. FDTD is suited to perform the type of calculations envisaged in this study. However, the strong need for a dense spatial and temporal discretization often limits the extent of the propagation region or the frequency range that can be considered. The use of a hybrid FDTD-PE (Parabolic equation method) could be a solution in a number of applications [13].

In this paper, the Ultra Weak Variational Formulation (UWVF) method in 2D is studied as an alternative. UWVF is related to the Finite Element Method (FEM), and can be further categorized as a Trefftz-type method. The UWVF method was firstly presented by O. Cessenat et al [14]. Later it was used to model the wave fields in biomedical ultrasonics [15, 16, 17]. The main reason to choose the UWVF method compared to FEM is the reduction by one of the dimensions of the integrals to be solved while constructing the system matrix. The basic FEM includes the integral in the computational cell, which usually occupies most of the computing time. The UWVF method only entails the integral along the cell boundary, leading to a reduced computational cost for solving the integrals. Another advantage of UWVF is the possibility to use large computational cells (up to two wavelengths [17]) without significant loss in accuracy. The reason for this is the split-up in plane wave basis functions that are a solution of the free field wave equation in the UWVF method.

In a time-domain method it is possible to obtain the response over a wide range of frequencies 
when working with a pulse-like source, and when applying a Fourier transform afterwards. The ability to obtain the required spectrum for typical traffic noise with a single simulation is clearly an advantage of FDTD. UWVF, on the other hand, is a frequency domain technique, and in principle a new calculation is needed for each frequency to be considered. However, it will be shown in this paper that the acoustic response over a broad frequency range can be calculated in a more efficient way.

A realistic evaluation of road traffic noise reducing measures needs the incorporation of multiple traffic lanes. The importance of this aspect is indicated e.g. in Ref. [18]. As an illustration, mixed traffic in two lanes induces the evaluation of 6 source positions according to the Harmonoise/Imagine source model [6]. In the UWVF, the matrices which form the final linear system do not depend on the location of the source. This means that changes in source position do not demand a full recalculation of the sound propagation problem. This is a benefit upon other numerical techniques like FDTD.

This paper is organized as follows. In the first part, the UWVF method is extended to the case of sound propagation through a porous medium. The Zwicker and Kosten rigid-frame porous medium model [19] is used to model sound propagation through the porous barrier. Different aspects of this numerical method are discussed, with focus on approaches to improve computational efficiency in typical traffic noise situations. In the second part, the UWVF method is validated. Firstly, a comparison is made with FDTD calculations for the case of sound propagation near a porous low-height barrier. Secondly, UWVF is checked with numerical calculations for sound propagation over porous ground in combination with a refracting atmosphere. In the third part, an assessment is made with UWVF on the use of porous low-height noise barriers in a realistic multi-lane traffic noise situation.

\section{The UWVF method}

\subsection{Equations for sound propagation in porous media}

In this paper, the Zwicker and Kosten phenomenological model [19] will be used to simulate sound propagation in and around the porous barriers. This is a rigid-frame porous model: only the air in between the particles is allowed to vibrate, while the constituting part of the porous medium is rigid. The choice of this particular porous medium model is mainly based on the fact that it can be readily implemented in the finite-difference time-domain method (as shown e.g. in $[11,20])$, to which UWVF calculations will be validated in Section 3.

Nevertheless, the Zwicker and Kosten phenomenological model is well-suited to model sound propagation through a porous medium. Only when there is interest in predicting the attenuation inside the porous medium at high sound frequencies and low flow resistivities, adaptions should be made as presented in [21]. Note, however, that the UWVF model is not restricted to a particular porous model, and other models can be implemented without additional difficulties.

Combining the Helmholtz equation and the Zwicker and Kosten rigid-frame porous model [19] 
leads to

$\nabla \cdot\left(\frac{1}{\rho} \nabla p\right)+\frac{\kappa^{2}}{\rho} p=f$

The wave number $\kappa$ and the density of the porous medium $\rho$ can be expressed as

$$
\begin{aligned}
& \kappa=\frac{\omega}{c} \sqrt{k_{s}+i \frac{R \varphi}{\omega \rho}}, \\
& \rho=\frac{\rho_{0} k_{s}}{\varphi},
\end{aligned}
$$

where $\omega$ is the angular frequency, $c$ is the speed of sound in air, $\rho_{0}$ is the density of air, $R$ is the flow resistivity, $k_{s}$ is the structure factor, $\varphi_{\text {is the porosity, and } i=\sqrt{-1}}$ is the imaginary unit. When $^{k_{s}}=1, \varphi=1$ and $R=0$, sound propagation in free air is described by equations (1)-(3).

Since UWVF is a volume-discretisation technique, the speed of sound can be location-dependent, and in this way a refracting atmosphere can be modeled by using the effective sound speed approach. The effective sound speed approach is useful to model refraction of sound by the presence of gradients in air temperature and wind speed in outdoor sound propagation calculations. Gradients in air temperature lead to gradients in the sound speed. Furthermore, the effect of wind on sound propagation can often be modeled by considering the horizontal component of the wind speed, leading to a virtual increase or decrease in the sound speed. In many applications, including sound propagation over noise barriers in wind [22], this is a good approach.

\subsection{Discretised problem}

For the two dimensional problem, the computational domain $\Omega$ is partitioned into a collection of disjoint triangular finite cells $\left\{K_{k}\right\}_{k=1}^{N}$. In Figure 1, two elements, $K_{k}$ and $K_{j}$ are shown to explain the notations. The outward unit normal vector on the boundary of $K_{k}$ is denoted by $n_{k}$. The boundary which connects the element $K_{k}$ to $K_{j}$ is $\Sigma_{k, j}$ and similarly $\Sigma_{j, k}$ connects $K_{j}$ to $K_{k}$. The interface $\Sigma_{k, j}$ has outgoing normal $n_{k}$, while the interface $\Sigma_{j, k}$ has outgoing normal $n_{j}$. If the boundary of the element $K_{k}$ is also an external boundary of the total domain $\Omega$, then this boundary is denoted as $\Gamma_{k}$.

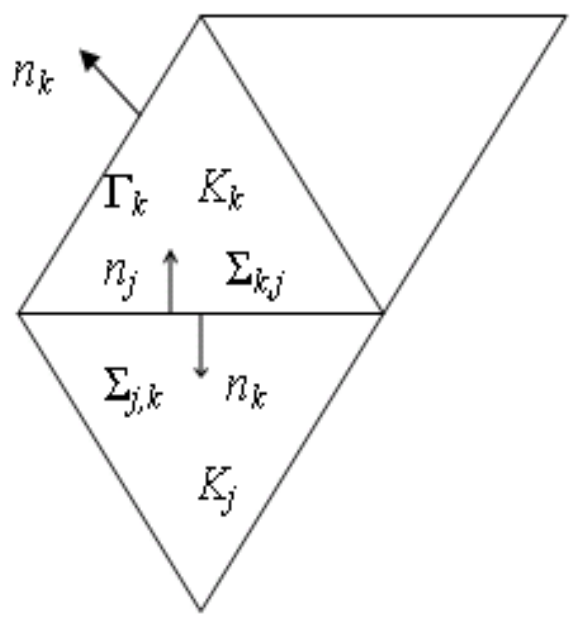


Figure 1: Three adjacent computational cells with notations.

The density and the wave number are assumed to be piecewise constants, so let $\rho_{k}=\left.\rho\right|_{K_{k}}$ and $\kappa_{k}=\left.\kappa\right|_{K_{k}}$. Therefore, the Helmholtz problem (1) in the domain $\Omega$ can be decomposed into a collection of sub-problems in the elements $K_{k}, k=1,2 \ldots N$

$\Delta p_{k}+\kappa_{k}^{2} p_{k}=f_{k} \quad$ in $K_{k}$,

where $f_{k}=f \cdot \rho_{k}$ is introduced.

On the interface between two neighboring cells, the continuity of velocity and the continuity of pressure must be satisfied $[14,15,16,17]$. For time harmonic waves, the continuity of velocity and the continuity of pressure between two neighboring cells $k$ and $j$ can be written as

$\begin{array}{ll}\frac{1}{\rho_{k}} \frac{i \omega}{i \omega-\frac{1}{\rho_{k}} R_{k}} \vec{n}_{k} \cdot \nabla p_{k}=-\frac{1}{\rho_{j}} \frac{i \omega}{i \omega-\frac{1}{\rho_{j}} R_{j}} \vec{n}_{j} \cdot \nabla p_{j} & \\ p_{k}=p_{j}, & , \quad \text { on } \Sigma_{k, j}\end{array}$

where the subscripts $k$ and $j$ denote the adjacent cells, $\vec{n}_{k}$ and $\vec{n}_{j}$ are the outgoing normal direction on the boundaries of the cells. For the non-porous medium, the flow resistivity $R_{k}$ and $R_{j}$ are equal to 0 , and equation (5) reduces to the equivalent formulas found in $[14,15,16,17]$.

\subsection{Perfectly matched layers}

Using efficient and accurate absorbing boundary conditions is of main importance in numerical models for outdoor sound propagation applications. The unbounded sound propagation region has to be truncated to a limited calculation domain. In this paper, the perfectly matched layer (PML) approach $[23,24]$ is chosen. The implementation of a PML in the UWVF method is based on extending the domain into a complex spatial coordinate space.

In the PML, the discretised Helmholtz equation can be written as

$$
\nabla \cdot\left(A_{k} \nabla p_{k}\right)+\kappa_{k}^{2} \eta_{k}^{2} p_{k}=f_{k} \eta_{k}^{2}, \quad \text { in } K_{k},
$$

where $A_{k}$ and $\eta_{k}$ are related to the complex spatial coordinates, and their definitions can be found in Ref. [15]. In the computational domain outside the PML region, $A_{k}=I$ and $\eta_{k}=1$ are chosen, where $I$ is the identity matrix, and equation (7) reduces to equation (4).

\subsection{Derivation of the $U W V F$}

Combining equations (5) and (6) gives:

$$
\begin{aligned}
& \frac{1}{\rho_{k}} \vec{n}_{k} \cdot\left(A_{k} \nabla p_{k}\right) \frac{i \omega}{i \omega-\frac{1}{\rho_{k}} R_{k}}-i \varsigma p_{k}=-\frac{1}{\rho_{j}} \vec{n}_{j} \cdot\left(A_{j} \nabla p_{j}\right) \frac{i \omega}{i \omega-\frac{1}{\rho_{j}} R_{j}}-i \varsigma p_{j} \\
& \frac{1}{\rho_{k}} \vec{n}_{k} \cdot\left(A_{k} \nabla p_{k}\right) \frac{i \omega}{i \omega-\frac{1}{\rho_{k}} R_{k}}+i \varsigma p_{k}=-\frac{1}{\rho_{j}} \vec{n}_{j} \cdot\left(A_{j} \nabla p_{j}\right) \frac{i \omega}{i \omega-\frac{1}{\rho_{j}} R_{j}}+i \varsigma p_{j} \quad \text { on }^{\Sigma_{k, j},} \quad \text { on }^{\Sigma_{k, j}} .
\end{aligned}
$$

Equations (8) and (9) are called the matching conditions. 
On the exterior boundary this is generalized to

$$
\left(\frac{1}{\rho_{k}} \vec{n}_{k} \cdot\left(A_{k} \nabla p_{k}\right) \frac{i \omega}{i \omega-\frac{1}{\rho_{k}} R_{k}}-i \varsigma p_{k}\right)=Q_{k}\left(-\frac{1}{\rho_{k}} \vec{n}_{k} \cdot\left(A_{k} \nabla p_{k}\right) \frac{i \omega}{i \omega-\frac{1}{\rho_{k}} R_{k}}-i \varsigma p_{k}\right)+g_{k}, \text { on } \Gamma_{k},
$$

where $\mathrm{Q}_{\mathrm{k}}$ is a complex constant and $\mathrm{g}_{\mathrm{k}}$ is a boundary source term. When $Q=1$ and $g_{k}=0$, the exterior boundary condition (10) becomes the rigid boundary condition. The parameter $\underline{S}$ must

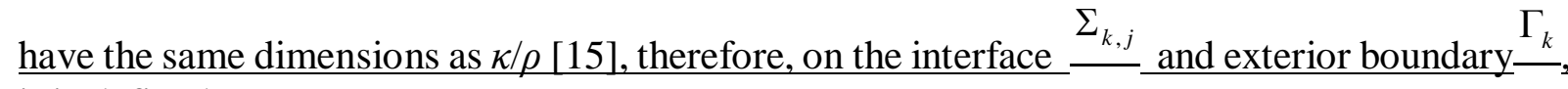
it is defined as

$\varsigma=\frac{1}{2}\left(\frac{\mathfrak{R}\left(\kappa_{k}\right)}{\rho_{k}}+\frac{\mathfrak{R}\left(\kappa_{j}\right)}{\rho_{j}}\right)$ and $\varsigma=\frac{\mathfrak{R}\left(\kappa_{k}\right)}{\rho_{k}}$,

respectively.

A new function $\chi_{k}$ is defined on the skeleton of the mesh

$$
\chi_{k}=-\frac{1}{\rho_{k}} \vec{n}_{k} \cdot\left(A_{k} \nabla p_{k}\right) \frac{i \omega}{i \omega-\frac{1}{\rho_{k}} R_{k}}-i \varsigma p_{k}, \quad, \quad 1 \leq k \leq N .
$$

It has been introduced in $[14,15,16,17]$ that $\chi_{k}$ satisfies the ultra weak variational formulation of the Helmholtz equation.

$$
\begin{aligned}
& \sum_{k=1}^{N} \int_{\partial \Omega_{k}} \frac{1}{\zeta} \chi_{k} \times \overline{\left(-\frac{1}{\rho_{k}} \vec{n}_{k} \cdot\left(\bar{A}_{k} \nabla\right)-i \varsigma\right) v_{k}}-\sum_{k=1}^{N} \sum_{j=1}^{N} \int_{\Sigma_{k, j}} \frac{1}{\zeta} \chi_{j} \times \overline{\left(\frac{1}{\rho_{k}} \vec{n}_{k} \cdot\left(\bar{A}_{k} \nabla\right)-i \varsigma\right) v_{k}} \\
- & \sum_{k=1}^{N} \int_{\Gamma_{k}} \frac{Q}{\zeta} \chi_{k} \times \overline{\left(\frac{1}{\rho_{k}} \vec{n}_{k} \cdot\left(\bar{A}_{k} \nabla\right)-i \varsigma\right) v_{k}}=\sum_{k=1}^{N} \int_{\Gamma_{k}} \frac{g_{k}}{\zeta} \times \overline{\left(\frac{1}{\rho_{k}} \vec{n}_{k} \cdot\left(\bar{A}_{k} \nabla\right)-i \varsigma\right) v_{k}-\sum_{k=1}^{N} \int_{\Omega_{k}} \frac{2 i}{\rho_{\mathrm{k}}} f_{k} \eta_{k}^{2} \bar{v}_{\mathrm{k}},}
\end{aligned}
$$

where the piecewise smooth function $v_{k}$ satisfies

$$
\nabla \cdot\left(A_{k} \nabla \overline{v_{k}}\right)+\kappa_{k}^{2} \eta_{k}^{2} \bar{v}_{k}=0, \quad \text { in } \Omega_{k} \text {. }
$$

The double summation in the second term of left hand side of equation (13) is limited to cells that share a common face ${ }^{\Sigma_{k, j}}$. The over bars stand for the complex conjugate.

\subsection{Expansion in plane waves}

In this paper, a local basis of plane waves is used for the expansion of the field within each triangular cell [25]

$\varphi_{k, m}=\left\{\begin{array}{lc}\exp \left(i \bar{\kappa}_{k} \vec{a}_{k, m} \cdot \overline{\vec{r}}^{\prime}\right) & \text { in } \Omega_{\mathrm{k}}, \\ 0 & \text { elsewhere, }\end{array}\right.$

where the index $k$ refers to the $k^{\text {th }}$ triangular cell, the index $m$ counts the basis functions within the cell, $\kappa_{k}$ is the complex wave number in the cell, $\vec{a}_{k, m}$ is the propagation direction of the $m^{\text {th }}$ basis function, and $\vec{r}^{\prime}$ is the spatial coordinate becoming complex within the PML. The 
function $\chi_{k}$ defined in equation (12) can be approximated as

$$
\chi_{k}^{a}=\left.\sum_{m=1}^{N_{k}}\left(\chi_{k, m}\left(-\frac{1}{\rho_{k}} \vec{n}_{k} \cdot\left(A_{k} \nabla\right)-i \varsigma\right) \varphi_{k, m}\right)\right|_{\partial \Omega_{k}}
$$

where $\chi_{k, m}$ is the unknown to be determined. Following the Galerkin method used in [14, 15, $16,17]$, we choose $v_{k}=\varphi_{k, \ell}$ as the test function. Substituting the plane wave basis functions into the discretised equation and using the same functions as test functions, a linear matrix system is obtained $[14,15]$ :

$(D-C) X=b$

where $X$ contains the unknowns, $D$ is a block diagonal matrix that comes from the first term of left hand side in equation (13) and contains blocks $D_{k}$ related to the $k^{\text {th }}$ cell; $C$ comes from the other two terms of left hand side in equation (13) and is a sparse block matrix coupling the solution in a single cell to adjacent cells and boundary conditions; $b$ is the only matrix that contains the information from the external boundary and sources.

\subsection{Solving the linear matrix system in broadband multi-source applications}

Wave based numerical methods are known to suffer from ill conditioning of the resulting matrix system. Huttunen et al. [17] have solved this problem by streamlining the condition number of each of the sub matrices $D_{k}$ by changing the number of basis functions in each cell. This iterative procedure can be lengthy and take most of the CPU-time in situations where the number of degrees of freedom is not extensive.

In road traffic noise applications, a wide range of frequencies has to be considered. The optimal number of basis functions for conditioning the matrices depends on the wavelength to cell size ratio and thus the computationally expensive iterative preconditioning operation needs to be repeated for every frequency of interest.

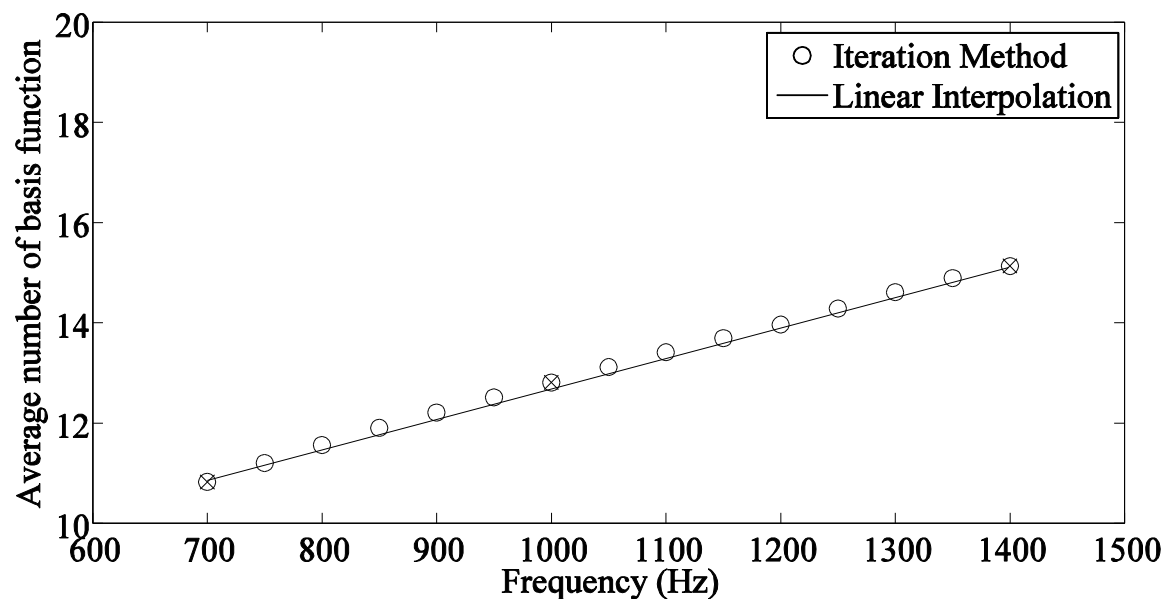

Figure 2: Average number of basis function in one grid calculated by an iteration procedure (open circles) 
and linear interpolation (solid line). The $\mathrm{x}$-signs indicate the 3 frequencies on which the linear interpolation is based.

To reduce calculation time, we use a linear interpolation to determine the number of basis functions for different frequencies.

Firstly, the optimal number of basis function for the upper, central and lower frequencies in an octave band is calculated. The choice of the number of basis function will determine the conditioning of the block matrix $D_{k}$. Ill-conditioned blocks can lead to poor approximations $[14,15]$. The 2-norm condition number can be a criterion to characterize the stability of the system defined by equation (17). An initial guess for the number of basis functions in each element is needed. Next, a searching procedure is required to find the largest number of basis functions by which the condition number of block matrix $\mathrm{D}_{k}$ is below a predetermined limit.

Secondly, a linear function is fitted to the relationship between the average number of basis functions per cell and the frequency. Finally, the required number of basis functions for the other frequencies constituting that octave band can be approximated by this linear function. An example is given in Figure 2. The comparison of the average number of basis functions in one grid calculated by iteration procedure and linear interpolation shows a good compliance.

The matrixes D, C and vector $\mathrm{b}$ can now be constructed avoiding the iterative procedure. Numerical tests indicated that by applying the linear interpolation, and by taking 21 sound frequencies to constitute the octave bands, the computational time can be shortened by $40 \%$ to $70 \%$, depending on the octave band considered. For high frequencies, assembling matrixes D and $\mathrm{C}$ occupies an equal amount of CPU time. The interpolation process can only limit the time used to assemble D. As a result, only $40 \%$ of the CPU time can be saved. For low frequencies, more time is used to assemble $\mathrm{D}$ when compared to $\mathrm{C}$. Therefore, $70 \%$ of $\mathrm{CPU}$ time can be gained by applying this interpolation. When different octave bands are considered, it is more advantageous to adapt the grid cell size to the wavelength instead of further interpolation. In this paper, the ratio between grid cell and the wavelength is chosen as 2 [15].

The numerical method can be further optimized in typical traffic noise situations with several source positions. Note that the matrixes D and C are only related to the geometrical properties of the triangular meshes, the medium density, and the frequency of the sound wave; the source position has no influence on the entries in $\mathrm{D}$ and $\mathrm{C}$. On the other hand, the vector $\mathrm{b}$ is derived from the source term and the boundary term, which are closely related to the source position. Therefore, if no parameters are changed except for the source position, the process to assemble matrixes $\mathrm{D}$ and $\mathrm{C}$ does not have to be repeated, which can reduce the computational cost by $90 \%$ for the cases studied in this paper.

\section{Numerical Validation}

\subsection{Sound propagation near a porous barrier}

In the first validation test, the UWVF method is compared to FDTD calculations for the case 
of sound propagation near a complex-shaped, low-height and porous noise barrier in a still and homogeneous atmosphere. An inverse $\Gamma$-shaped porous barrier of $1 \mathrm{~m}$ high and $1 \mathrm{~m}$ wide is considered, as show in Figure 3. Two dimensional calculations are performed.

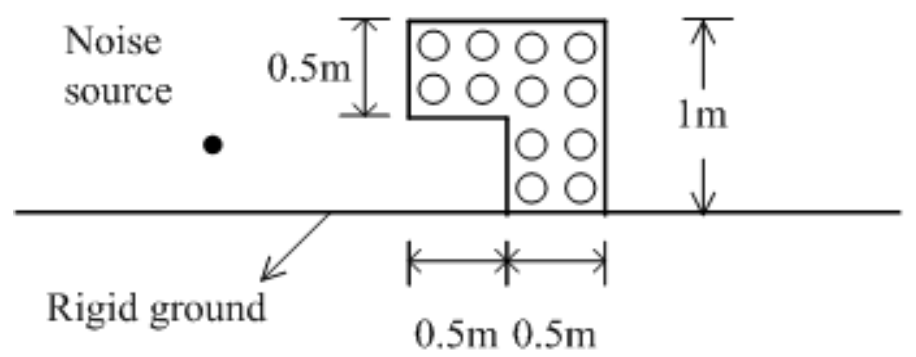

Figure 3: Geometry of the porous noise barrier for the validation example.

The computational domain is $4 \mathrm{~m}$ wide and $3 \mathrm{~m}$ high. Perfectly matched layers are used to border the simulation domain. The frequency of the sound wave under study is $1000 \mathrm{~Hz}$. The noise source is present at $1 \mathrm{~m}$ in front of the noise barrier and at $0.3 \mathrm{~m}$ above the rigid ground. The porosity, structure factor and flow resistivity of the barrier are chosen as 0.4, 1.35 and $50 \mathrm{k} \mathrm{Pa} \cdot \mathrm{s} / \mathrm{m}^{2}$, respectively. Although no particular material is aimed at, such a set of parameters could be representative for uncompacted earth [26,21]. In the UWVF method, the largest allowed mesh size of $0.68 \mathrm{~m}$ is used, which is twice the wave length which equals $0.34 \mathrm{~m}$ when using a speed of sound of $340 \mathrm{~m} / \mathrm{s}$. In total, 8666 unknowns are considered in the UWVF computation. For the FDTD calculation, a much larger number of unknowns are used. Since the FDTD method serves as a reference calculation here, a very fine spatial discretisation step of $0.01 \mathrm{~m}$ is used. This means that for the sound frequency under study, more than 30 computational cells per wavelength are present. This is far beyond the common practice of 10 computational cells per wavelength, and will lead to a strong reduction of possible phase errors. The Courant number is set to 1. For the porous barrier, an implementation of the Zwikker and Kosten model [19] is used as well in FDTD. As in the UWVF method, perfectly matched layers are used to model an unbounded propagation domain. A detailed description of the FDTD implementation can be found in Ref. [11].

In Figure 4, the insertion loss fields are shown as calculated with UWVF and FDTD. All complex aspects of the sound field near this barrier are very well predicted by the UWVF method, showing its validity in this non-trivial sound propagation problem. The difference between the IL fields calculated with UWVF and FDTD is depicted in Fig. 5. At most locations, the differences range from $-2 \mathrm{~dB}$ to $+2 \mathrm{~dB}$. In zones where pronounced interference of sound waves occurs, the largest differences are found. 

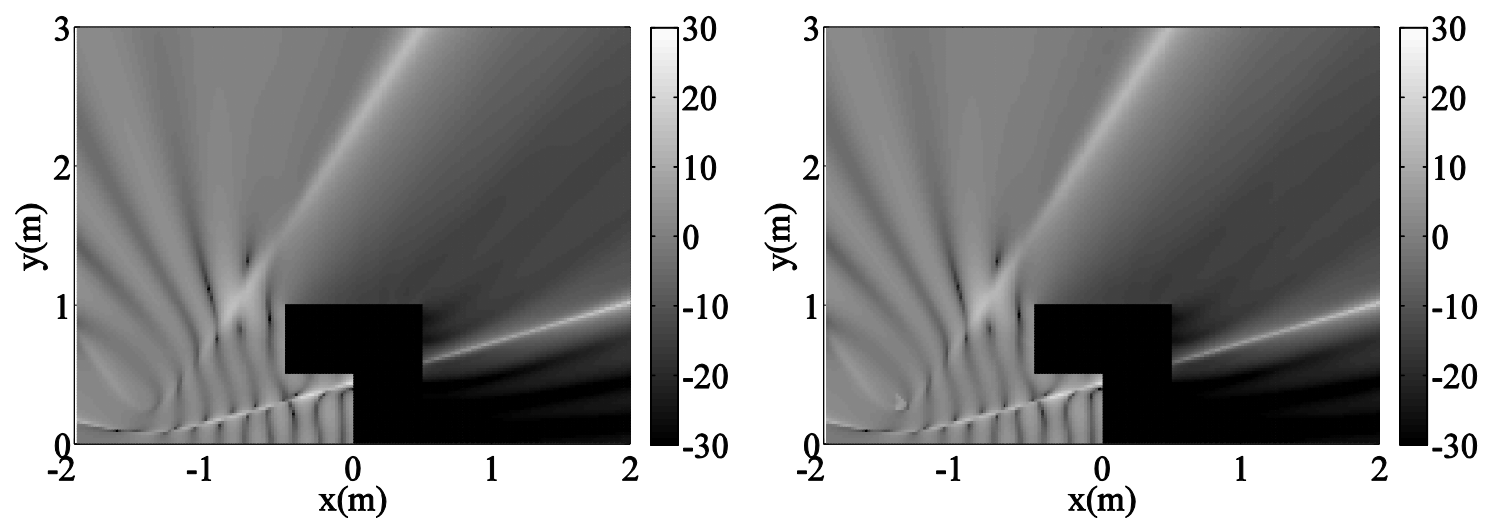

Figure 4: Comparison of the noise barrier insertion loss at a single sound frequency of $1000 \mathrm{~Hz}$. On the left, calculations with FDTD are depicted, on the right UWVF is used.

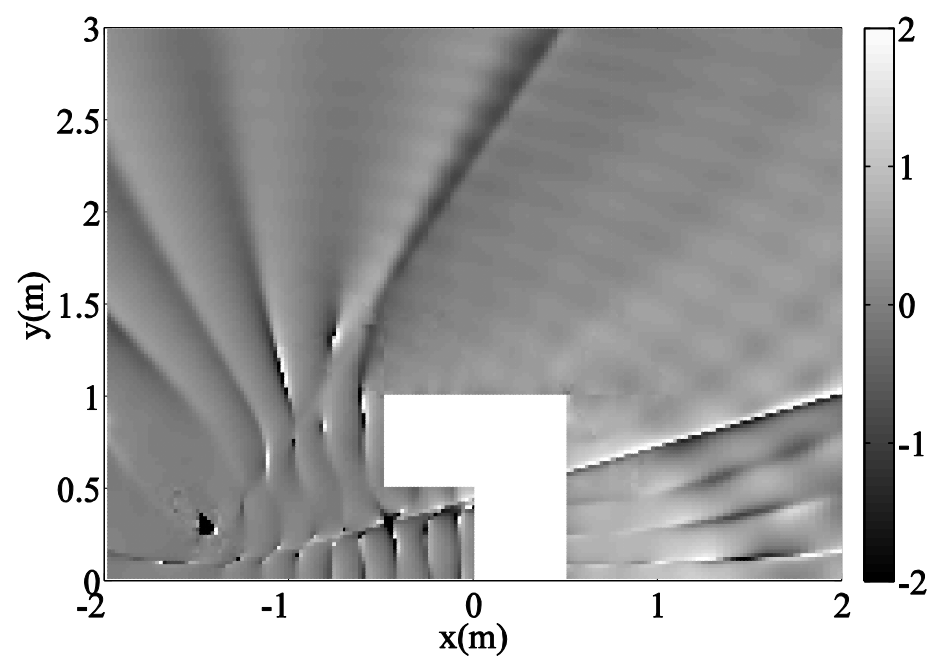

Figure 5: Difference between insertion loss fields of FDTD and UWVF as shown in Figure 4.

\subsection{Sound propagation over porous ground in a refracting atmosphere}

In the second validation test, sound propagation over porous ground in a refracting atmosphere is studied. A source is located at a height of $0.5 \mathrm{~m}$ above the ground; the receiver is at a height of $1.5 \mathrm{~m}$. A two dimensional calculation is performed. The example chosen here is similar to the one presented in Ref. [27]. The distance between the source and receiver is 5 m. Sound frequencies between $100 \mathrm{~Hz}$ and $1000 \mathrm{~Hz}$ are considered. Results are expressed relative to free field sound propagation. In Figure 6 and Figure 7, a homogeneous and downwardly refracting atmosphere is considered, respectively. In case of the non-homogeneous atmosphere, a linear increase in the speed of sound, with a gradient of $3 / \mathrm{s}$ is used. The numerical results show that in case of downward refraction, the ground dip is somewhat more pronounced, and a shift towards lower frequencies is observed. At $100 \mathrm{~Hz}$, the difference between both models amounts up to $0.5 \mathrm{~dB}$. The UWVF method is in general in good agreement with FDTD calculations in both situations, showing its validity in a non-homogeneous sound propagation medium as well. 


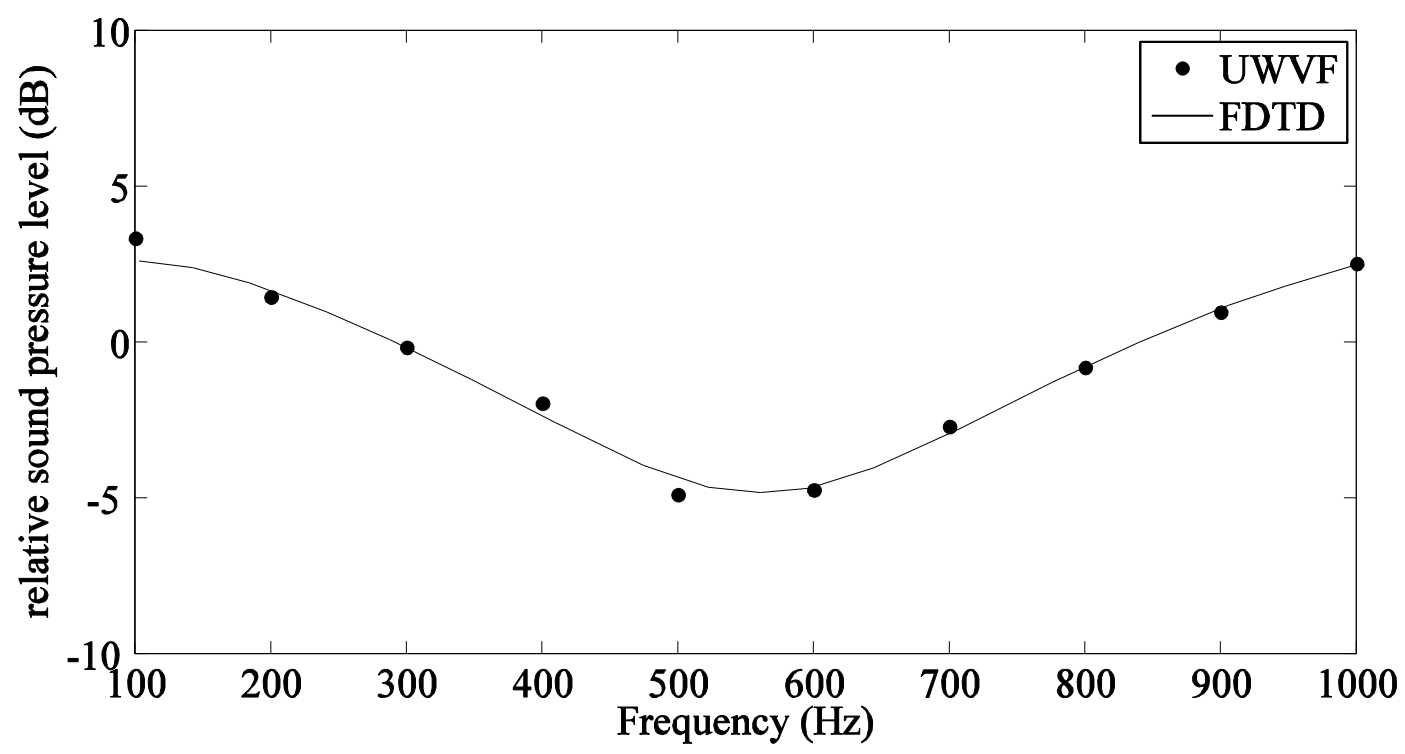

Figure 6: Spectra of the relative sound pressure level in a non-refracting atmosphere. The porosity of the ground equals 0.3 , the structure factor equals 3.0 and flow resistivity equals $10 \mathrm{k} P a \cdot s / \mathrm{m}^{2}$.

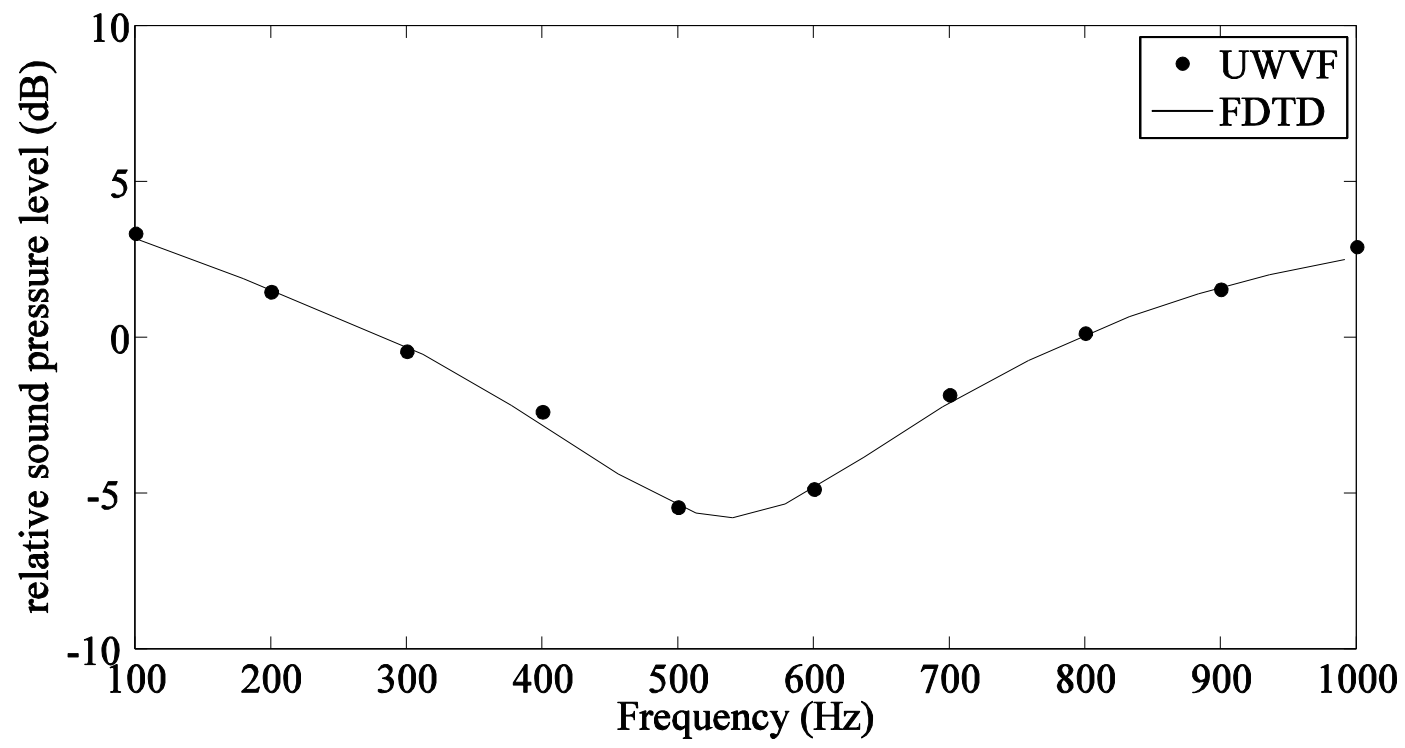

Figure 7: Spectra of the relative sound pressure level in a downwardly refracting atmosphere. A linearly increasing sound speed profile is modeled, with a gradient of $3 / \mathrm{s}$. The porosity of the ground equals 0.3 , the structure factor equals 3.0 and flow resistivity equals $10 \mathrm{k} \mathrm{Pa} \cdot \mathrm{s} / \mathrm{m}^{2}$.

\section{Numerical assessment of porous barriers in realistic traffic noise situations}

In this section, the effect of porous and rigid low-height barriers along a two-lane road is studied as shown in Figure 8. The width of each traffic lane is $4 \mathrm{~m}$. When mixed traffic (including both light and heavy vehicles) is modeled, sound emitted by 6 point sources must be considered following the Harmonoise/Imagine road traffic source model [6]. The point sources are located in the middle of the traffic lanes. Points P3 and P6 (at a height $0.01 \mathrm{~m}$ ) are 
for the tire/road noise interaction of both light and heavy cars. P2 and P5 (at a height of 0.30 $\mathrm{m}$ ) represent the engine noise sources of light vehicles, while P1 and P4 (at a height of $0.75 \mathrm{~m}$ ) represent the engine noise sources of heavy vehicles.

Two dimensional calculations are performed. This means that the barrier has a constant cross-section, and that a coherent line source is modeled. However, the Harmonoise/Imagine road traffic source model was developed for a point source. To overcome this discrepancy, the equivalence between sound pressure levels, expressed relative to free field sound propagation, of a coherent line source and a point source is assumed [13].

Both the street surface and pavement are modeled as rigid. A homogeneous and windless atmosphere is assumed. Given the low barrier height and the short propagation distances between the road traffic noise sources and the passengers, screen-induced refraction of sound is most likely very limited.

Various noise barriers were considered in this traffic noise assessment. These are shown in Figure 9. Both the height and the width of all barriers is $1 \mathrm{~m}$. Five inverse $\Gamma$-shaped barriers were tested, with different properties. The first one, barrier (a), is completely rigid. Barriers (b) till (f) are porous and have the same porosity and structure factor, of 0.4 and 1.35 , respectively. Barriers (b) and (c) have a flow resistivity of $50 \mathrm{k} \mathrm{Pa} \cdot \mathrm{s} / \mathrm{m}^{2}$, while (d) and (e) have a lower flow resistivity of $10 \mathrm{k} \mathrm{Pa} \cdot \mathrm{s} / \mathrm{m}^{2}$. Furthermore, a rigid thin inner barrier is inserted in barriers (c) and (e) to prevent possible transmission through the porous barrier. Barrier (f) has a rectangular shape and a flow resistivity of $50 \mathrm{k} \mathrm{Pa} \cdot \mathrm{s} / \mathrm{m}^{2}$.

Three regions of interest were defined behind the barrier where passengers could appear. The upper region extends $5 \mathrm{~m}$ behind the noise barrier, at a height between $1.5 \mathrm{~m}$ and $2 \mathrm{~m}$. The middle one, at heights between $1.0 \mathrm{~m}$ and $1.5 \mathrm{~m}$, and the lower region of interest is located between a height of $0.5 \mathrm{~m}$ and $1 \mathrm{~m}$. Results are expressed as the average insertion loss for total A-weighted traffic noise in a given region of interest. The full traffic noise spectrum [6] is modeled by including the octave bands with central frequencies ranging from $63 \mathrm{~Hz}$ till $2000 \mathrm{~Hz}$. For each octave band, 21 equally-spaced frequencies were sufficient for calculating the octave band level. Three vehicles speeds were considered, namely 30, 50 and $70 \mathrm{~km} / \mathrm{h}$.

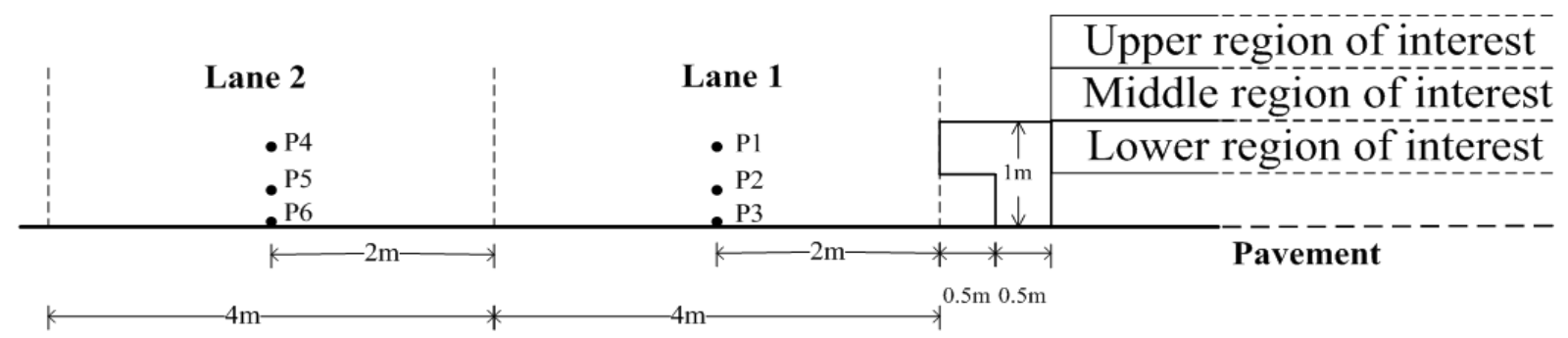

Figure 8: Multi-lane traffic noise situation under study for the assessment of low-height noise barriers 


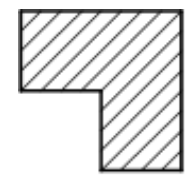

(a)

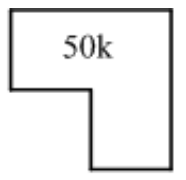

(b)

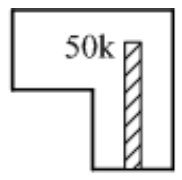

(c)

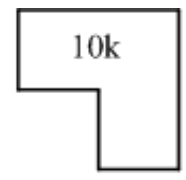

(d)

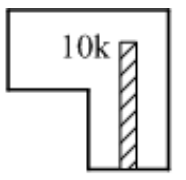

(e)

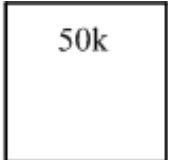

(f)

Figure 9: Configurations studied: (a) rigid noise barrier (RNB); (b) porous noise barrier, flow resistivity $50 \mathrm{k} \mathrm{Pa} \cdot \mathrm{s} / \mathrm{m}^{2}$ (PNB50); (c) porous noise barrier with inner rigid barrier, flow resistivity $50 \mathrm{k} \mathrm{Pa} \cdot \mathrm{s} / \mathrm{m}^{2}$ (PNB50_RI); (d) porous noise barrier, flow resistivity 10k Pa $\cdot \mathrm{s} / \mathrm{m}^{2}$ (PNB10); (e) porous noise barrier with inner rigid barrier, flow resistivity $10 \mathrm{k} \mathrm{Pa} \cdot \mathrm{s} / \mathrm{m}^{2}\left(\mathrm{PNB} 10 \_\mathrm{RI}\right)$; (e) rectangular porous noise barrier $(1 \mathrm{~m} \times 1 \mathrm{~m})$, flow resistivity $50 \mathrm{k} \mathrm{Pa} \cdot \mathrm{s} / \mathrm{m}^{2}$ (PNB50_REC). For all porous noise barriers, the porosity is 0.4 and the structure factor is 1.35 .

In Figure 10, the total traffic noise insertion loss is shown at different vehicles speeds $(30,50$, and $70 \mathrm{~km} / \mathrm{h}$ ), for the different barrier configurations considered. Four traffic noise situations are considered, namely a light vehicle in lane 1, a light vehicle in lane 2, a heavy vehicle in lane 1 , and a heavy vehicle in lane 2 . The upper region of interest is considered here, since this is the typical ear height of an adult person. Values for the insertion loss range from 2.5 dBA till 9 dBA.

Lane use is an important parameter. The presence of either a light or heavy vehicle in lane 1 gives a higher insertion loss than when the vehicle is present in lane 2. Lane choice has a bigger influence for light vehicles than for heavy vehicles. The presence of vehicles in lane 2 leads to more noise generation sources that can contribute directly, without diffraction, to the upper region.

It is further observed that the insertion loss is higher for light vehicles. For these, high frequencies contribute more to the total traffic noise levels. Diffraction around an obstacle is less pronounced for higher frequencies, and furthermore, the absorption at the barrier top is larger. With increasing vehicle speed, higher frequencies and rolling noise become more dominant, and larger insertion losses are observed. This effect is more pronounced for either light or heavy vehicles present in lane 1 . For heavy traffic in lane 2, this trend is not observed, and even a very small decrease in insertion loss with increasing vehicle speed can be found for some barriers.

When comparing the different screens, it is observed that the porous barriers give in all cases an improvement in shielding relative to the rigid one. At maximum, an increase in insertion of $2 \mathrm{dBA}$ is observed for light vehicles. For heavy vehicles, an increased shielding up to $1 \mathrm{dBA}$ is found.

The use of a lower flow resistivity of $10 \mathrm{k} \mathrm{Pa} \cdot \mathrm{s} / \mathrm{m}^{2}$ gives a somewhat worse performance than in case of $50 \mathrm{k} \mathrm{Pa} \cdot \mathrm{s} / \mathrm{m}^{2}$, especially for light vehicles in lane 1 . In this specific traffic noise situation, the difference may amount up to $1.2 \mathrm{dBA}$. For heavy traffic, the influence of the flow resistivities considered in this numerical evaluation is very limited.

The presence of a rigid thin inner barrier has only a limited effect for the flow resistivities 
considered. At $50 \mathrm{k} \mathrm{Pa} \cdot \mathrm{s} / \mathrm{m}^{2}$, there is sufficient damping when sound propagates through the barrier. As a consequence, transmission through the barrier is subordinate to diffracting waves over it. At a flow resistivity of $10 \mathrm{k} \mathrm{Pa} \cdot \mathrm{s} / \mathrm{m}^{2}$, the leaking of acoustical energy through the barrier has some influence on the total traffic noise levels. Preventing this transmission path by placing such an inner barrier improves shielding only to a small extent. It is further observed that a rectangular screen does not give any improvement relative to the inversely gamma-shaped screen with similar porous medium parameters.
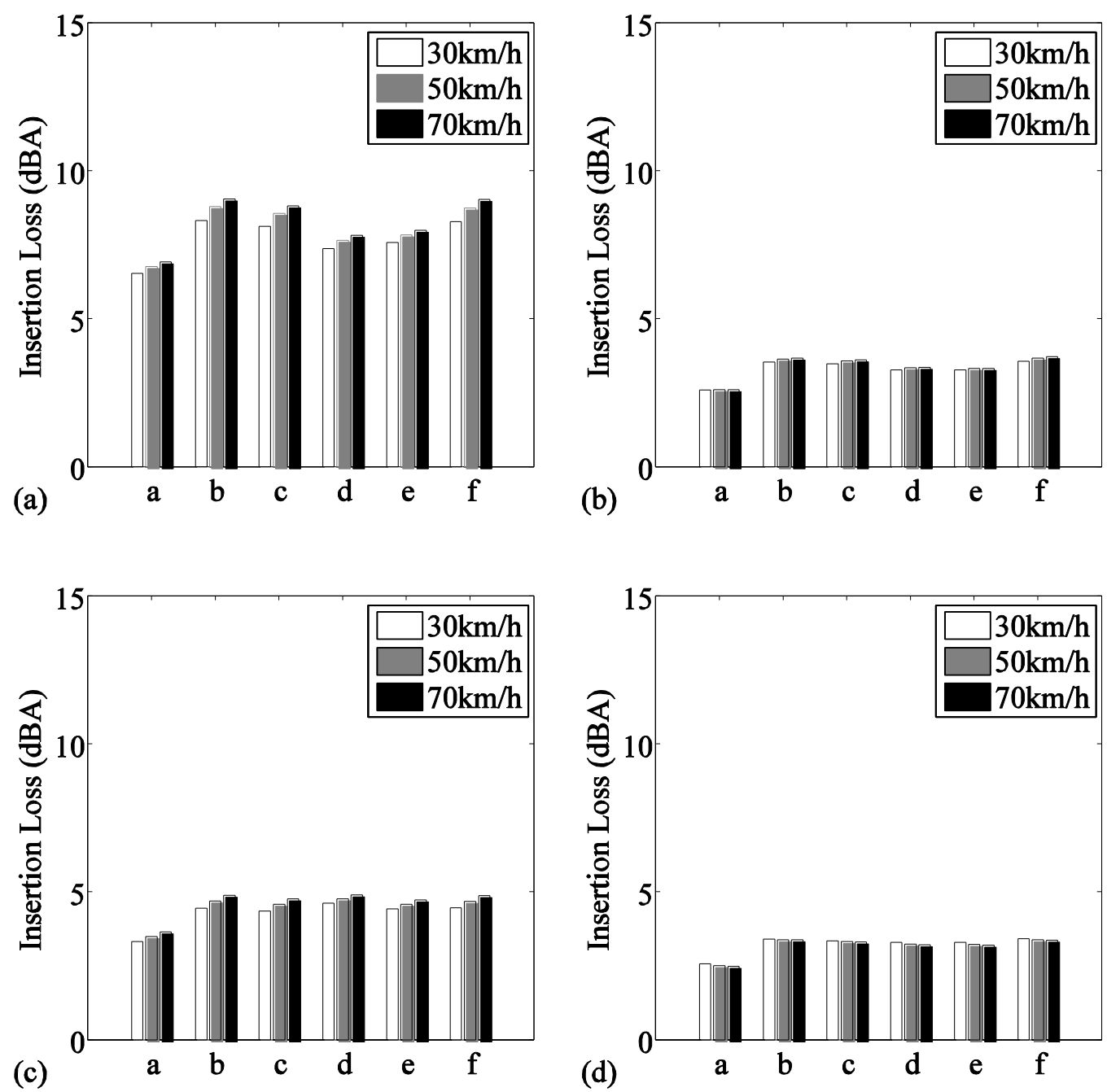

Figure 10: Average insertion loss in the upper region of interest: a) Light vehicle in lane 1; b) Light vehicle in lane 2; c) Heavy vehicle in lane 1; d) Heavy vehicle in lane 2.

When analyzing different octave bands in more detail, as is shown in Figure 11 for vehicle speeds of $50 \mathrm{~km} / \mathrm{h}$ and sound propagation to the upper region, we clearly see the complex nature of this sound propagation problem. In case of a light vehicle in lane one, an increase in insertion loss with increasing frequency is observed. For higher frequencies, the differences between the various noise barrier configurations increase. For the other source locations, a more complex behavior is observed. For both a light and heavy vehicle in lane 2, the insertion loss is very similar. The main difference here is the presence of the engine noise source at a 
height of $0.75 \mathrm{~m}$ instead of $0.30 \mathrm{~m}$. The difference in engine noise height is much more important when situated close to the barrier. Note that the sound paths leading to destructive interference in the reference situation (i.e. rigid ground without obstacles) might be prevented by the presence of a barrier. This could lead to a very low insertion loss, as is observed for heavy traffic in lane 1 for the octave band with central frequency $500 \mathrm{~Hz}$. The insertion loss of the gamma shape compared to the full barrier is larger in the $63 \mathrm{~Hz}$ octave band due to an internal resonance in the cavity. Its performance is slightly worse only at a few frequencies where insertion loss is high anyhow. An advantage of the gamma shaped barrier is that the amount of material needed for construction is lower.
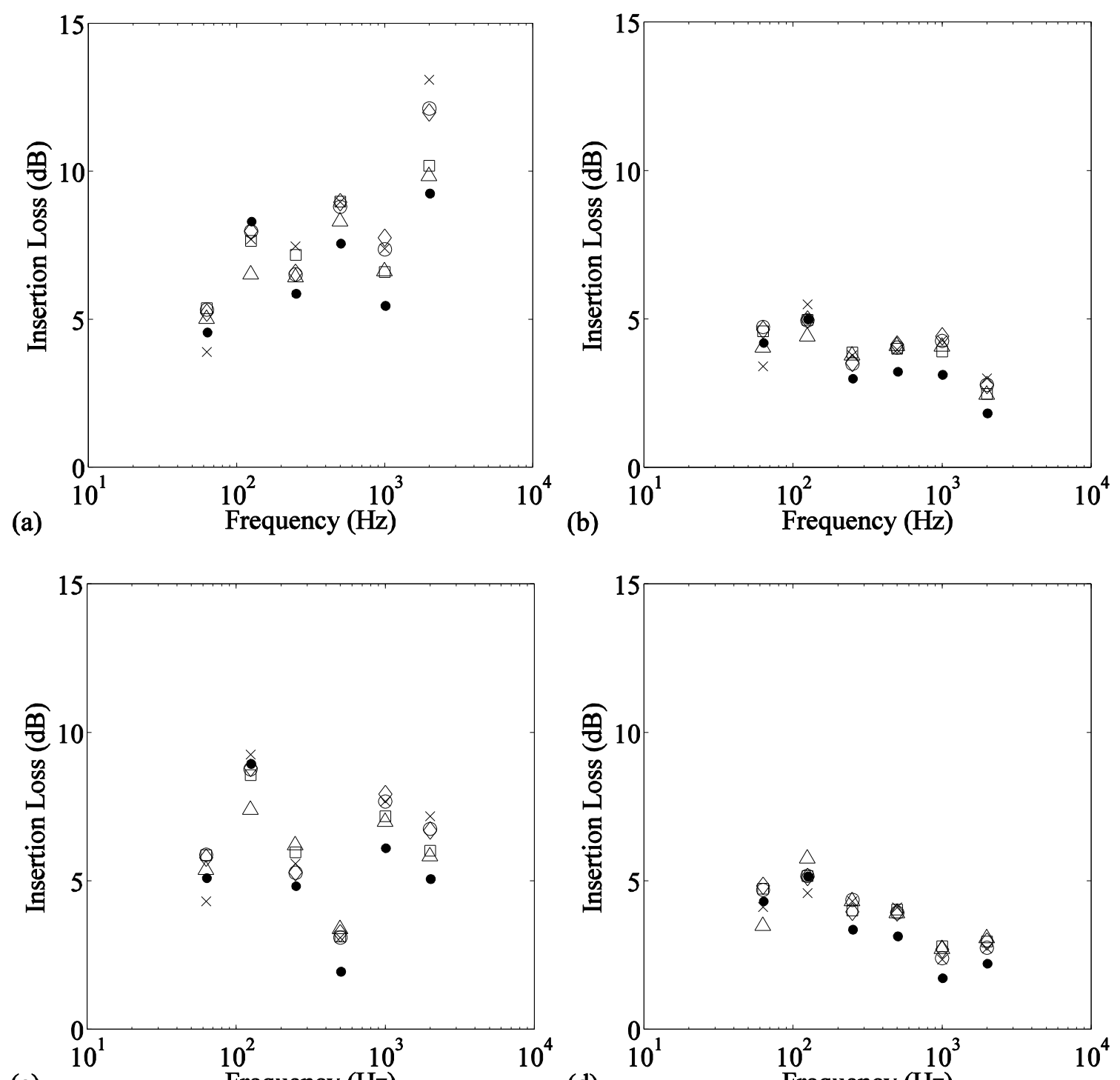

(c)

Frequency $(\mathrm{Hz})$

Figure 11: Average insertion loss $(\mathrm{dB})$ in the upper region of interest for different octave bands. Vehicle speed is $50 \mathrm{~km} / \mathrm{h}$. $\bullet$ : rigid noise barrier; $\diamond$ : porous noise barrier, flow resistivity $50 \mathrm{k} \mathrm{Pa} \cdot \mathrm{s} / \mathrm{m}^{2}$; ०: porous noise barrier with rigid bar inside, flow resistivity $50 \mathrm{k} \mathrm{Pa} \cdot \mathrm{s} / \mathrm{m}^{2} ; \Delta$ : porous noise barrier, flow resistivity $10 \mathrm{k}$ $\mathrm{Pa} \cdot \mathrm{s} / \mathrm{m}^{2}$; $\square$ : porous noise barrier with rigid bar inside, flow resistivity $10 \mathrm{k}$ Pa $\cdot \mathrm{s} / \mathrm{m}^{2} ; \times$ : rectangular porous noise barrier, flow resistivity 50k Pa.s $/ \mathrm{m}^{2}$ : a) Light vehicle in lane one; b) Light vehicle in lane two; c) Heavy vehicle in lane one; d) Heavy vehicle in lane two.

The shielding in the lower regions is significantly higher (see Table 1 and Figure 12 and Figure 13). The area in the receiver zone with direct sound contributions decreases, and only 
diffracted and transmitted sound energy is found there. In the middle region, values of the insertion loss range from $5.1 \mathrm{~dB}$ up to $13.0 \mathrm{~dB}$, when considering all barriers, lane choices, vehicle types, and vehicle speeds. In the lower region of interest, these values range from 9.9 $\mathrm{dB}$ to $17.9 \mathrm{~dB}$. Lane choice has a smaller effect than in the upper region of interest.

In the middle and lower regions of interest, the presence of a thin rigid barrier inside the $10 \mathrm{k}$ $\mathrm{Pa} \cdot \mathrm{s} / \mathrm{m}^{2}$ barrier leads to a more significant increase in insertion loss of up to $1 \mathrm{~dB}$. For the $50 \mathrm{k}$ $\mathrm{Pa} \cdot \mathrm{s} / \mathrm{m}^{2}$ barrier, the effect of the inner rigid barrier stays limited. In the lower region, the typical increase of noise barrier shielding with frequency as for common highway noise barriers is found as shown in Figure 13.

Table 1: Average insertion loss $(\mathrm{dB})$ in the three regions of interest. Vehicle speed is 30,50 , and $70 \mathrm{~km} / \mathrm{h}$. LV denotes light vehicle, HV denotes heavy vehicle. L1 denotes traffic lane 1, L2 denotes traffic lane 2.

\begin{tabular}{|c|c|c|c|c|c|c|c|c|c|c|}
\hline & \multicolumn{3}{|l|}{ RNB } & \multicolumn{3}{|l|}{ PNB50 } & \multicolumn{3}{|c|}{ PNB50_RI } \\
\hline & & $30 \mathrm{~km} / \mathrm{h}$ & $50 \mathrm{~km} / \mathrm{h}$ & $70 \mathrm{~km} / \mathrm{h}$ & $30 \mathrm{~km} / \mathrm{h}$ & $50 \mathrm{~km} / \mathrm{h}$ & $70 \mathrm{~km} / \mathrm{h}$ & $30 \mathrm{~km} / \mathrm{h}$ & $50 \mathrm{~km} / \mathrm{h}$ & $70 \mathrm{~km} / \mathrm{h}$ \\
\hline \multirow[t]{3}{*}{$\mathrm{LV}$ in $\mathrm{L1}$} & Upper & 6.54 & 6.77 & 6.93 & 8.32 & 8.79 & 9.05 & 8.13 & 8.56 & 8.82 \\
\hline & Middle & 9.65 & 10.20 & 10.49 & 11.56 & 12.51 & 13.01 & 11.38 & 12.27 & 12.74 \\
\hline & Lower & 13.25 & 14.66 & 15.32 & 14.63 & 16.64 & 17.69 & 14.56 & 16.48 & 17.47 \\
\hline \multirow[t]{3}{*}{$\mathrm{LV}$ in $\mathrm{L} 2$} & Upper & 2.59 & 2.61 & 2.61 & 3.54 & 3.64 & 3.67 & 3.48 & 3.58 & 3.61 \\
\hline & Middle & 6.42 & 6.78 & 6.93 & 7.81 & 8.39 & 8.66 & 7.72 & 8.27 & 8.54 \\
\hline & Lower & 11.41 & 12.52 & 13.04 & 12.36 & 13.91 & 14.69 & 12.28 & 13.76 & 14.50 \\
\hline \multirow[t]{3}{*}{$\mathrm{HV}$ in $\mathrm{L} 1$} & Upper & 3.32 & 3.49 & 3.65 & 4.45 & 4.69 & 4.89 & 4.35 & 4.58 & 4.77 \\
\hline & Middle & 5.54 & 5.67 & 5.81 & 6.81 & 7.01 & 7.21 & 6.68 & 6.87 & 7.06 \\
\hline & Lower & 10.04 & 10.29 & 10.50 & 11.36 & 11.73 & 12.02 & 11.20 & 11.55 & 11.82 \\
\hline \multirow[t]{5}{*}{$\mathrm{HV}$ in $\mathrm{L} 2$} & Upper & 2.57 & 2.51 & 2.48 & 3.41 & 3.38 & 3.38 & 3.35 & 3.32 & 3.31 \\
\hline & Middle & 5.14 & 5.13 & 5.14 & 6.37 & 6.45 & 6.52 & 6.26 & 6.32 & 6.38 \\
\hline & Lower & 9.85 & 10.23 & 10.47 & 10.98 & 11.52 & 11.89 & 10.88 & 11.40 & 11.74 \\
\hline & & \multicolumn{3}{|l|}{ PNB10 } & \multicolumn{3}{|c|}{ PNB10_RI } & \multicolumn{3}{|c|}{ PNB50_REC } \\
\hline & & $30 \mathrm{~km} / \mathrm{h}$ & $50 \mathrm{~km} / \mathrm{h}$ & $70 \mathrm{~km} / \mathrm{h}$ & $30 \mathrm{~km} / \mathrm{h}$ & $50 \mathrm{~km} / \mathrm{h}$ & $70 \mathrm{~km} / \mathrm{h}$ & $30 \mathrm{~km} / \mathrm{h}$ & $50 \mathrm{~km} / \mathrm{h}$ & $70 \mathrm{~km} / \mathrm{h}$ \\
\hline \multirow[t]{3}{*}{$\mathrm{LV}$ in $\mathrm{L} 1$} & Upper & 7.37 & 7.65 & 7.82 & 7.58 & 7.84 & 8.00 & 8.28 & 8.75 & 9.04 \\
\hline & Middle & 10.24 & 10.97 & 11.34 & 10.74 & 11.38 & 11.71 & 11.41 & 12.43 & 12.99 \\
\hline & Lower & 13.22 & 14.7 & 15.35 & 13.98 & 15.51 & 16.24 & 14.3 & 16.48 & 17.64 \\
\hline \multirow[t]{3}{*}{$\mathrm{LV}$ in $\mathrm{L} 2$} & Upper & 3.28 & 3.35 & 3.36 & 3.27 & 3.32 & 3.32 & 3.57 & 3.68 & 3.72 \\
\hline & Middle & 7.15 & 7.69 & 7.94 & 7.41 & 7.86 & 8.07 & 7.84 & 8.45 & 8.76 \\
\hline & Lower & 11.17 & 12.31 & 12.86 & 12.11 & 13.38 & 13.99 & 12.18 & 13.79 & 14.63 \\
\hline \multirow[t]{3}{*}{$\mathrm{HV}$ in L1 } & Upper & 4.62 & 4.77 & 4.90 & 4.42 & 4.58 & 4.73 & 4.46 & 4.68 & 4.87 \\
\hline & Middle & 7.14 & 7.30 & 7.46 & 6.77 & 6.92 & 7.08 & 6.76 & 6.95 & 7.15 \\
\hline & Lower & 11.04 & 11.43 & 11.72 & 11.24 & 11.55 & 11.79 & 11.17 & 11.57 & 11.88 \\
\hline \multirow[t]{3}{*}{$\mathrm{HV}$ in $\mathrm{L} 2$} & Upper & 3.3 & 3.24 & 3.21 & 3.30 & 3.23 & 3.20 & 3.42 & 3.38 & 3.37 \\
\hline & Middle & 5.98 & 6.04 & 6.09 & 6.12 & 6.14 & 6.18 & 6.36 & 6.42 & 6.48 \\
\hline & Lower & 10.14 & 10.58 & 10.87 & 10.73 & 11.17 & 11.47 & 10.98 & 11.53 & 11.91 \\
\hline
\end{tabular}



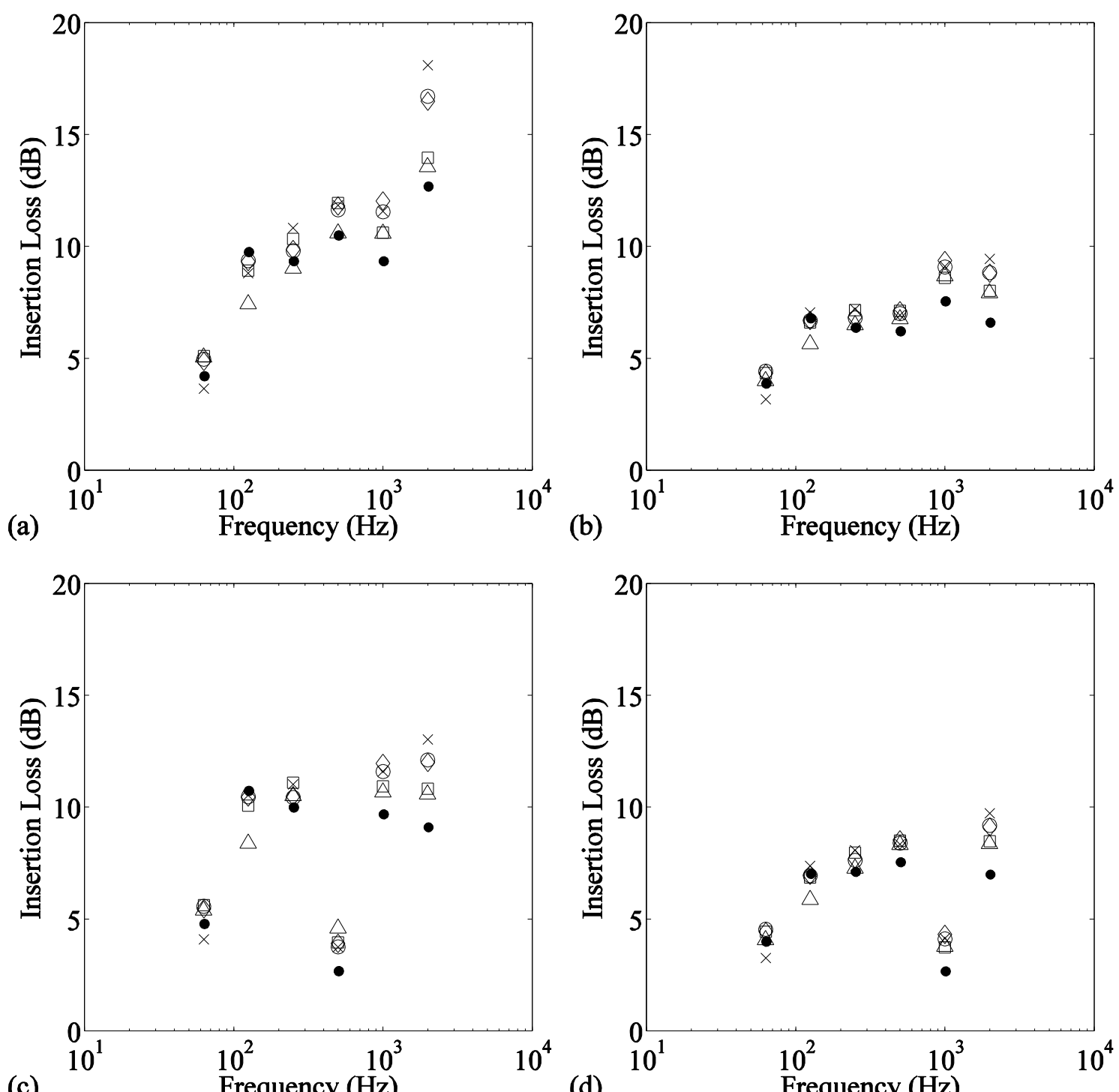

Figure 12: Average insertion loss $(\mathrm{dB})$ in the middle region of interest for different octave bands. Vehicle speed is $50 \mathrm{~km} / \mathrm{h}$. For definition of symbols see Figure 11: a) Light vehicle in lane one; b) Light vehicle in lane two; c) Heavy vehicle in lane one; d) Heavy vehicle in lane two.
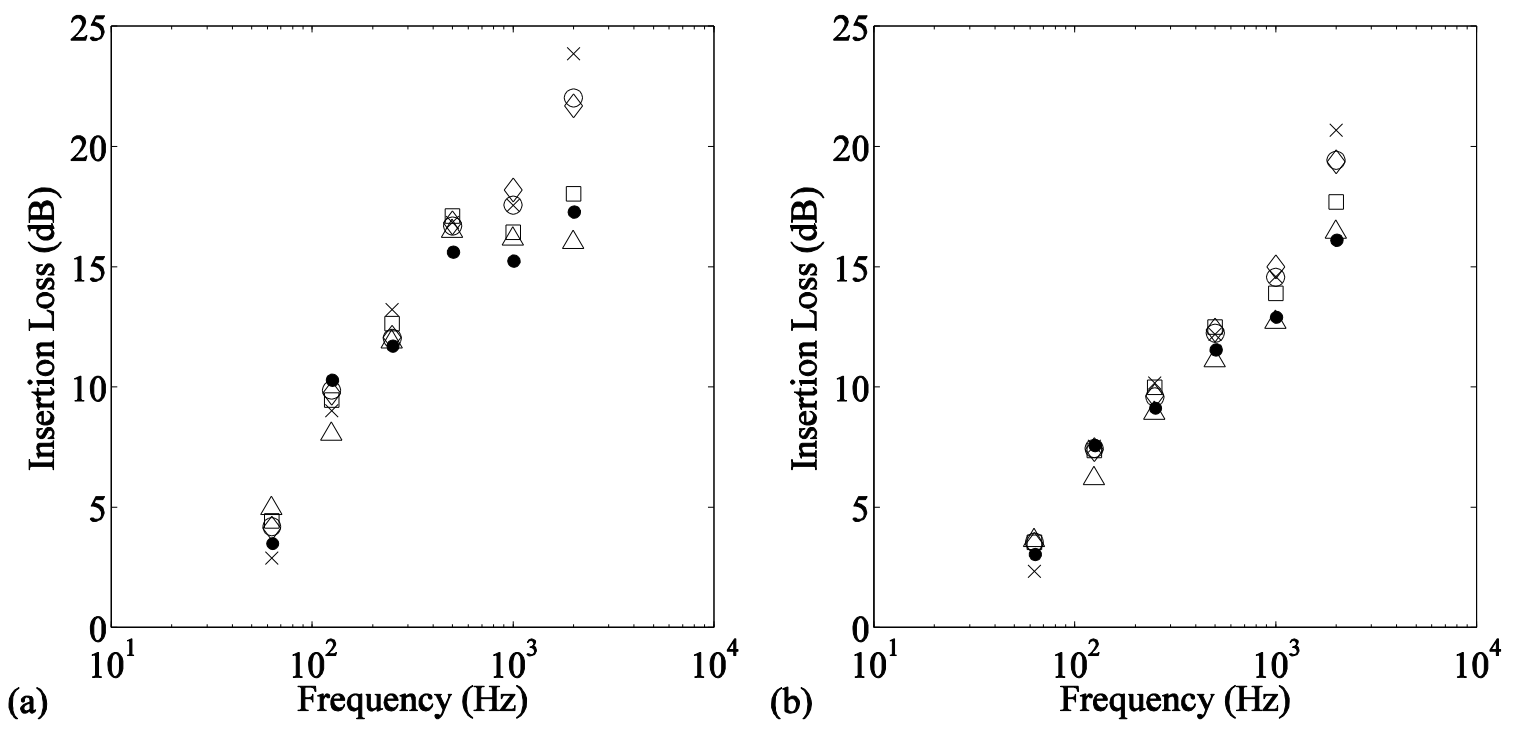

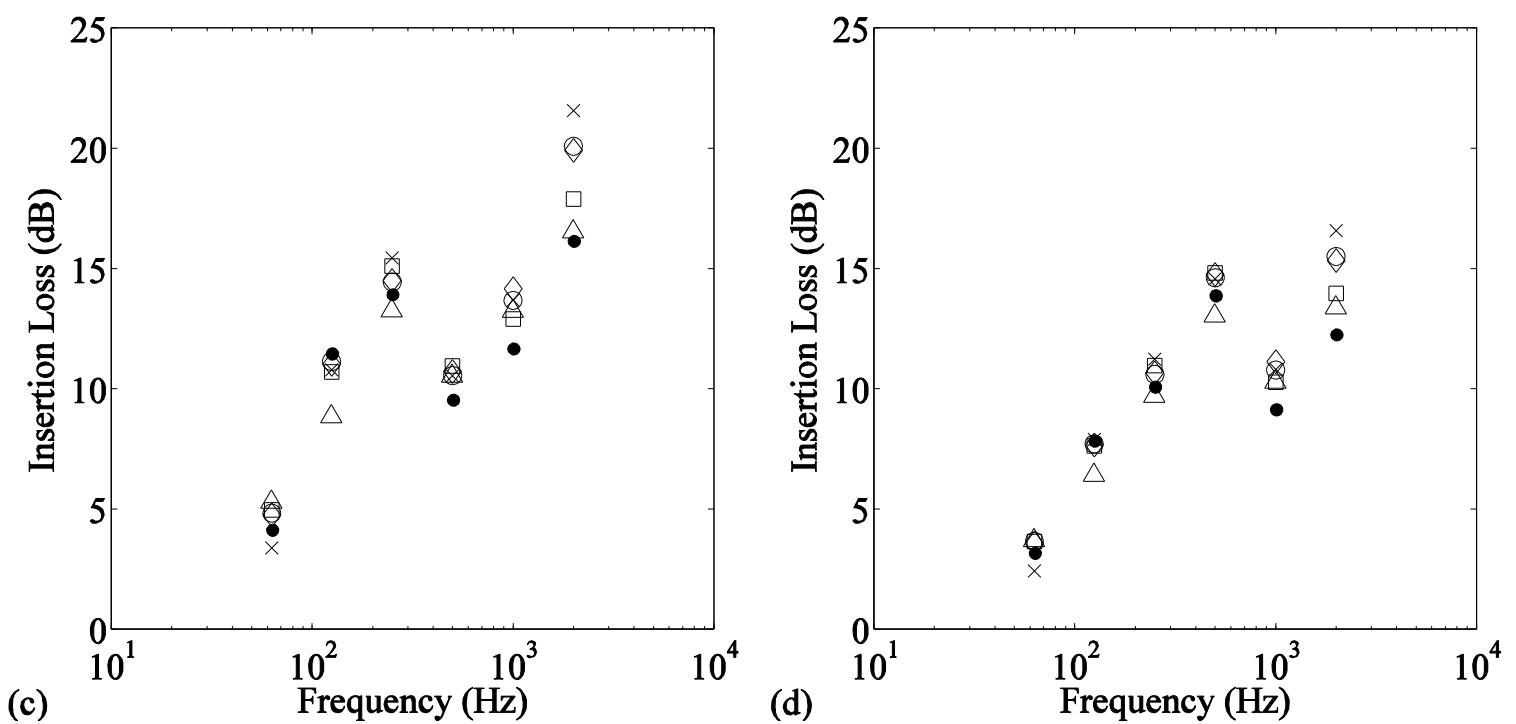

Figure 13: Average insertion loss $(\mathrm{dB})$ in the lower region of interest for different octave bands. Vehicle speed is $50 \mathrm{~km} / \mathrm{h}$. For definition of symbols see Figure 11: a) Light vehicle in lane one; b) Light vehicle in lane two; c) Heavy vehicle in lane one; d) Heavy vehicle in lane two.

\section{Conclusion}

In this paper, the ultra weak variational formulation (UWVF) approach in 2D is used to study the effect of semi-transparent road traffic noise barriers of limited height for passengers at the pavement. This numerical method is extended to simulate sound propagation through a porous medium, based on the Zwikker and Kosten porous rigid-frame model. Since the UWVF method is a volume-discretisation technique, the effective sound speed approach could be used to model sound propagation in a refracting atmosphere.

Focus is on an efficient approach to calculate noise levels in multi-lane road traffic noise situations. Finding the number of basis functions for each frequency is a time-consuming task. It is approached by 3 initial evaluations per octave band. For the other frequencies constituting that band, the number of basis functions could be linearly interpolated without loss of accuracy. Furthermore, it was shown that the exact source location only influences some of the matrices forming the system to be solved. The presence of different source locations which is typical in mixed multi-lane road traffic can therefore be solved in an efficient way.

The UWVF method was validated successfully by comparison with $2 \mathrm{D}$ finite-difference time-domain (FDTD) calculations. Two important outdoor sound propagation cases were considered, namely sound propagation near a (porous) noise barrier, and sound propagation above porous ground in a refracting atmosphere.

An assessment is made of the use of various porous low-height noise barriers of $1 \mathrm{~m}$ height near multi-lane road traffic noise sources. Focus is on the zone were the human ear might be present. The Harmonoise/Imagine road traffic source model is used to predict total traffic noise power levels. Depending on the type of traffic and the lane choice, values for the average insertion loss in a zone with heights between $1.5 \mathrm{~m}$ to $2 \mathrm{~m}$ (and for a distance of up to 
$5 \mathrm{~m}$ behind the noise barrier) range from $2.5 \mathrm{dBA}$ till $9 \mathrm{dBA}$. The vehicle speeds considered were 30,50 , and $70 \mathrm{~km} / \mathrm{h}$. It can therefore be concluded that although such zones are not completely shielded, significant reductions in the sound pressure level are nevertheless found. The effect of vehicle speed on the insertion loss was shown to be very limited. At lower receiver levels, the values for the insertion loss are much higher, and may amount up to 18 dBA.

Porous barriers can improve noise shielding up to $2 \mathrm{~dB}$ for light vehicles, when compared to geometrically identical rigid noise barriers. The flow resistivity of the porous medium was shown to be an important property. However, a detailed optimization of the porous medium properties is not considered in this study. For more transparent low-height noise barriers, the use of an inner rigid barrier leads to an improvement in shielding, mainly at lower receiver heights.

\section{References}

[1] D. Ouis: Annoyance from road traffic noise: a review. Journal of Environmental Psychology 21 (2001) 101-120.

[2] R. Klaeboe: Are adverse impacts of neighbourhood noisy areas the flip side of quiet area benefits? Applied Acoustics 68 (2007) 557-575.

[3] K. V. Horoshenkov, D. C. Hothersall, S. E. Mercy: Scale modeling of sound propagation in a city street canyon. Journal of Sound and Vibration 223 (1999) 795-819.

[4] P. J. Thorsson: Optimisation of low-height noise barriers using the equivalent sources method. Acta Acustica united with Acustica 86 (2000) 811-820.

[5] M. Baulac, J. Defrance, P. Jean, F. Minard: Efficiency of noise protections in urban areas: predictions and scale model measurements. Acta Acustica united with Acustica 92 (2006) $530-539$.

[6] H. G. Jonasson: Acoustical source modelling of road vehicles. Acta Acustica united with Acustica 93 (2007) 173-184.

[7] T. Van Renterghem, D. Botteldooren: Reducing the acoustical façade load from road traffic with green roofs. Building and Environment 44 (2009) 1081-1087.

[8] T. Van Renterghem, D. Botteldooren: Numerical evaluation of sound propagating over green roofs. Journal of Sound and Vibration 317 (2008) 781-799.

[9] S. J. Martin, D. C. Hothersall: Numerical modeling of median road traffic noise barriers. Journal of Sound and Vibration 251 (2002) 671-681.

[10] E. M. Salomons: Reduction of the performance of a noise screen due to screen-induced wind-speed gradients. Numerical computations and wind tunnel experiments. Journal of Acoustical Society of America 105 (1999) 2287-2293.

[11] T. Van Renterghem, D. Botteldooren: Numerical simulation of the effect of trees on downwind noise barrier performance. Acta Acustica united with Acustica 89 (2003) 764-778.

[12] R. Blumrich, D. Heimann: A linearized Eulerian sound propagation model for studies of complex meteorological effects. Journal of the Acoustical Society of America 112 (2002) 446-455.

[13] T. Van Renterghem, E. Salomons, D. Botteldooren: Efficient FDTD-PE model for sound propagation in situations with complex obstacles and wind profiles. Acta Acustica united with Acustica 91 (2005) 671-679.

[14] O. Cessenat, B. Despres: Application of an ultra weak variational formulation of elliptic PDEs to the two-dimensional Helmholtz problem. SIAM Journal of Numerical Analysis 
35 (1998) 255-299.

[15] T. Huttunen: The ultra weak variational formulation for ultrasound transmission problems. PhD Thesis, Kuopio University, 2004.

[16] T. Huttunen, J. P. Kaipio, P. Monk: The perfectly matched layer for the ultra weak variational formulation of the 3D Helmholtz equation. International Journal for Numerical Methods in Engineering 61 (2004) 1072-1092.

[17] T. Huttunen, P. Monk J. P. Kaipio: Computational aspects of the ultra-weak variational formulation. Journal of Computational Physics 182 (2002) 27-46.

[18] E. Walerian, R. Janczur, M. Meissner, M. Czechowicz: Influence of vehicle noise emission directivity on sound level distribution in a canyon street. Part II: experimental verification. Applied Acoustics 67 (2006) 659-679.

[19] C. Zwicker, C. Kosten: Sound absorbing materials. Elsevier, New York, 1949.

[20] E. M. Salomons, R. Blumrich, D. Heimann: Eulerian time-domain model for sound propagation over a finite-impedance ground surface: Comparison with frequency-domain models. Acustica united with Acta acustica 88 (2002) 483-492.

[21] K. Wilson, V. Ostashev, S. Collier, N. Symons, D. Aldridge, D. Marlin: Time-domain calculations of sound interactions with outdoor ground surfaces. Applied Acoustics 68 (2007) 173-200.

[22] Blumrich R, Heimann D: Numerical estimation of atmospheric approximation effects in outdoor sound propagation modeling. Acta Acustica united with Acustica 90 (2004) 24-37

[23] J. P. Berenger: A perfectly matched layer for the absorption of electromagnetic waves. Journal of Computational Physics 114 (1994) 185-200.

[24] F. Ihlenburg: Finite Element Analysis of Acoustic Scattering. Springer, 1998.

[25] O. Cessenat, B. Despres: Using plane waves as base functions for solving time harmonic equations with the ultra weak variational formulation. Journal of Computational Acoustics 11 (2003) 227-238.

[26] K. Attenborough: Ground parameter information for propagation modeling. Journal of the Acoustical Society of America 92 (1992) 418-427.

[27] E. M. Salomons, R. Blumrich, D. Heimann: Eulerian time-domain model for sound propagation over a finite-impedance ground surface. Comparison with frequency-domain models. Acustica united with Acta acustica 88 (2002) 483-492. 